\title{
PRIMER REGISTRO DE LA FAMILIA AGAONIDAE (HYMENOPTERA: CHALCIDOIDEA) EN LA FAUNA VIVIENTE DE LA HISPANIOLA
}

\author{
Candy Ramírez Pérez
}

Instituto de Investigaciones Botánicas y Zoológicas Prof. Rafael M. Moscoso, Universidad Autónoma de Santo Domingo (UASD), Santo Domingo, República Dominicana. ramirez.candy@hotmail.com

\section{RESUMEN}

Se registra la presencia de la familia Agaonidae (Hymenoptera: Chalcidoidea) para la República Dominicana en base a ejemplares colectados recientemente. Hasta el momento se han identificado los géneros Pegoscapus Cameron, 1906 y Tetrapus Mayr, 1885.

Palabras clave: Agaonidae, Hymenoptera, avispas de los higos, Ficus, República Dominicana, Hispaniola.

Title: First record of family Agaonidae (Hymenoptera: Chalcidoidea) in the extant fauna of Hispaniola.

\section{ABSTRACT}

The family Agaonidae (Hymenoptera, Chalcidoidea) is reported for the Dominican Republic based on specimens collected recently. Until now the genera Pegoscapus Cameron, 1906 and Tetrapus Mayr, 1885, have been identified.

Key words: Agaonidae, Hymenoptera, fig wasps, Ficus, Dominican Republic, Hispaniola.

Las "avispas de los higos" pertenecen a la familia Agaonidae (Chalcidoidea), que forman parte de uno de los grupos más diversos de Hymenoptera, siendo mayormente insectos pequeños (1-3 mm de tamaño). La familia Agaonidae consta de 78 géneros, algunos de los cuales son muy importantes para la polinización de las plantas del género Ficus Linnaeus, 1753, desarrollándose así un mutualismo obligado entre ellos. Las hembras polinizan las flores transportando polen desde el sicono donde nacen hasta otros siconos, a los que penetran, poniendo sus huevos en flores femeninas de estilo corto. Una vez que pone sus huevos, la avispa muere.

La importancia de los agaónidos radica en que sin ellos los Ficus no serían polinizados, lo que produciría un efecto negativo, ya que muchos animales frugívoros se alimentan de los higos producidos por estos árboles (Rasplus y Soldati, 2006). El género Ficus está ampliamente distribuido en los trópicos del mundo, incluyendo unas 800 especies, de las cuales 125 se encuentran en América (Cardona et al., 2007). En la República Dominicana hay 18 especies registradas, ocho nativas y 10 introducidas (Acevedo-Rodríguez y Strong, 2012). Se pensaba que por cada especie de Ficus había una especie de avispa Agaonidae para polinizarla, pero se han registrado otras avispas que no polinizan la planta sino que usan los frutos donde está el polinizador y otras que usan los frutos de la planta para lograr el desarrollo de sus larvas sin contribuir en nada con la polinización ni estar presente el polinizador (Cardona et al., 2007).

Hasta el momento, en la Hispaniola la familia Agaonidae solo había sido registrada de la fauna fósil preservada en el ámbar dominicano. Peñalver et al. (2006) describen tres especies 


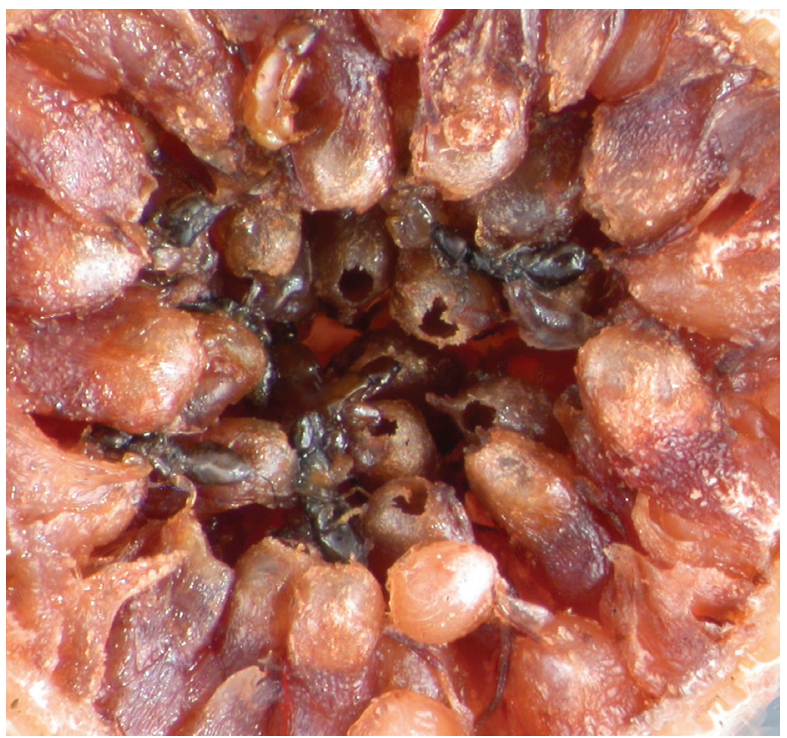

Figura 1. Individuos de Agaonidae dentro de sicono colectado en Santo Domingo, D. N.

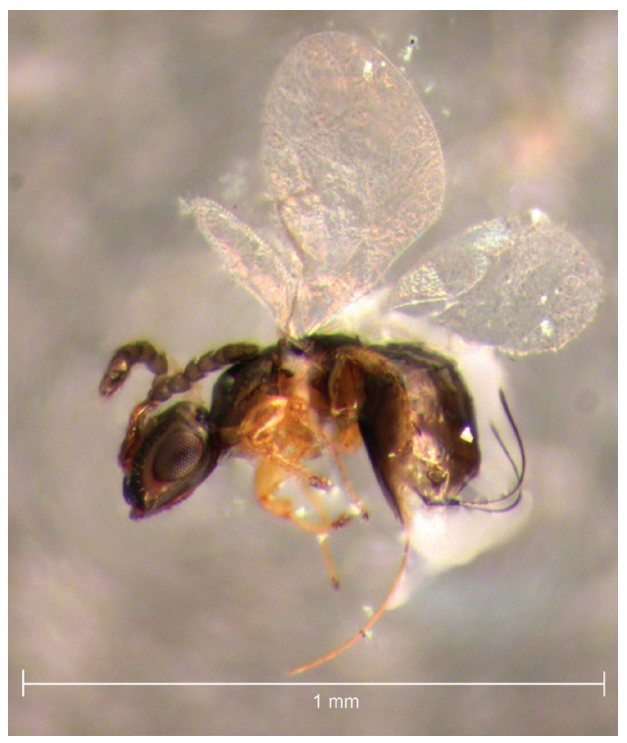

Figura 2. Hembra de Tetrapus sp.

correspondientes a los géneros Pegoscapus y Tetrapus. En la base de datos en línea de insectos del Caribe del Departamento de Entomología del Museo de Zoología Comparada de la Universidad de Harvard (http://insects.oeb.harvard.edu/caribbean/search.htm) se incluyen dos ejemplares colectados en Punta Cana en el 2004 por Brian Farrell y otros investigadores; los especímenes fueron identificados hasta la categoría de familia, pero hasta el momento no se había realizado ningún registro oficial de los mismos. Ambos ejemplares están depositados en la Colección Entomológica de Punta Cana y exhiben la siguiente información en la etiqueta: Dominican Republic, La Altagracia, Higuey, Reserva Ecológica Punta Cana, 27 March - 4 April 2004, Farrell, Guerrero \& Nunez. ID: 30869, hembra; 30876, macho.

En el 2012 se inició un estudio con la finalidad de conocer la composición de la fauna de avispas asociadas a los Ficus en la Hispaniola, donde se colectaron alrededor de 10 siconos de diferentes especies de Ficus en las provincias de Barahona, San Pedro de Macorís, Samaná, San Juan y el Distrito Nacional, registrándose la presencia de las avispas en cada uno de los siconos colectados (Fig. 1). La presencia de Agaonidae en la República Dominicana abre las puertas para innumerables e interesantes trabajos sobre las relaciones ecológicas entre los Ficus y sus polinizadores, así como nuevos registros de especies y localidades. Se determinaron dos géneros, usando la clave dicotómica de Rasplus y Soldati (2006). En San Pedro de Macorís se encontró el género Pegoscapus y en el Distrito Nacional se encontró el género Tetrapus (Fig. 2). Hasta el momento, ambos géneros son exclusivos del continente americano, Pegoscapus cuenta con 52 especies y Tetrapus con ocho especies (Universal Chalcidoidea Database, 2012).

\section{AGRADECIMIENTOS}

Al Instituto de Investigaciones Botánicas y Zoológicas (IIBZ) en la persona de su Directora, la entomóloga Ruth Bastardo; a Santo Navarro, por sus enseñanzas sobre el grupo Hymenoptera; a Ángela Guerrero por sus motivaciones y a Daniel Perez-Gelabert por los atinados comentarios hechos al manuscrito. 


\section{LITERATURA CITADA}

Acevedo-Rodríguez, P. y M. T. Strong. 2012. Catalogue of Seed Plants of the West Indies. Smithsonian Contributions to Botany, 98: 1,192 pp.

Cardona, W., P. Chacón De Ulloa y G. Kattan. 2007. Avispas no polinizadoras asociadas a Ficus andicola (Moraceae) en la Cordillera Central de Colombia. Revista Colombiana de Entomología 33 (2): 165-170.

Noyes, J. S. Junio 2012. Universal Chalcidoidea Database. World Wide Web electronic publication. http://www.nhm.ac.uk/chalcidoids.

Peñalver, E., M. S. Engel y D. A. Grimaldi. 2006. Fig wasps in Dominican amber (Hymenoptera: Agaonidae). American Museum Novitates 3541: 1-16.

Rasplus, J. Y. y L. Soldati. 2006. Familia Agaonidae, 683-698 pp. En: F. Fernández y M. J. Sharkey, eds. Introducción a los Hymenoptera de la Región Neotropical. Editorial Universidad Nacional de Colombia, Bogotá.

[Recibido: 10 de abril, 2013. Aceptado para publicación: 24 de abril, 2013] 